Lecture Notes in Economics and Mathematical Systems 676

Frédéric Amblard

Francisco J. Miguel

Adrien Blanchet

Benoit Gaudou Editors

Advances

in Artifial

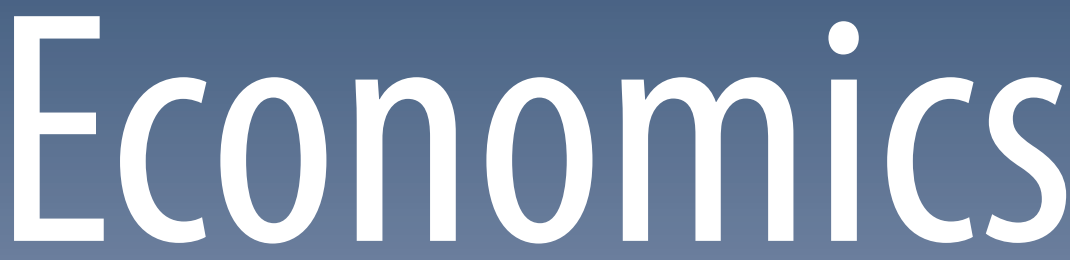




\section{Lecture Notes in Economics and Mathematical Systems}

Founding Editors:

M. Beckmann

H.P. Künzi

Managing Editors:

Prof. Dr. G. Fandel

Fachbereich Wirtschaftswissenschaften

Fernuniversität Hagen

Hagen, Germany

Prof. Dr. W. Trockel

Murat Sertel Institute for Advanced Economic Research

Istanbul Bilgi University

Istanbul, Turkey

and

Institut für Mathematische Wirtschaftsforschung (IMW)

Universität Bielefeld

Bielefeld, Germany

Editorial Board:

H. Dawid, D. Dimitrov, A. Gerber, C-J. Haake, C. Hofmann, T. Pfeiffer,

R. Slowiński, W.H.M. Zijm 
More information about this series at http://www.springer.com/series/300 
Frédéric Amblard • Francisco J. Miguel • Adrien Blanchet $\bullet$ Benoit Gaudou

Editors

\section{Advances in Artificial Economics}

照 Springer 


\section{Editors}

Frédéric Amblard

Adrien Blanchet

Benoit Gaudou

University Toulouse 1 Capitole

Toulouse

France

\author{
Francisco J. Miguel \\ Laboratory for Socio-Historical \\ Dynamics (LSDS) \\ Universitat Autònoma de Barcelona \\ Cerdanyola del Vallès \\ Spain
}

ISSN 0075-8442

ISBN 978-3-319-09577-6

DOI 10.1007/978-3-319-09578-3

Springer Cham Heidelberg New York Dordrecht London
ISSN 2196-9957 (electronic)

ISBN 978-3-319-09578-3 (eBook)

Library of Congress Control Number: 2014955224

(c) Springer International Publishing Switzerland 2015

This work is subject to copyright. All rights are reserved by the Publisher, whether the whole or part of the material is concerned, specifically the rights of translation, reprinting, reuse of illustrations, recitation, broadcasting, reproduction on microfilms or in any other physical way, and transmission or information storage and retrieval, electronic adaptation, computer software, or by similar or dissimilar methodology now known or hereafter developed. Exempted from this legal reservation are brief excerpts in connection with reviews or scholarly analysis or material supplied specifically for the purpose of being entered and executed on a computer system, for exclusive use by the purchaser of the work. Duplication of this publication or parts thereof is permitted only under the provisions of the Copyright Law of the Publisher's location, in its current version, and permission for use must always be obtained from Springer. Permissions for use may be obtained through RightsLink at the Copyright Clearance Center. Violations are liable to prosecution under the respective Copyright Law.

The use of general descriptive names, registered names, trademarks, service marks, etc. in this publication does not imply, even in the absence of a specific statement, that such names are exempt from the relevant protective laws and regulations and therefore free for general use.

While the advice and information in this book are believed to be true and accurate at the date of publication, neither the authors nor the editors nor the publisher can accept any legal responsibility for any errors or omissions that may be made. The publisher makes no warranty, express or implied, with respect to the material contained herein.

Printed on acid-free paper

Springer is part of Springer Science+Business Media (www.springer.com) 


\title{
Trade-In Programs in the Context of Technological Innovation with Herding
}

\author{
Paolo Pellizzari, Elena Sartori, and Marco Tolotti
}

\section{Introduction}

In the Summer of 2013, Apple launched a new updating campaign in the US to entice old iPhone 4 users to switch to the new iPhone 5 (see Rampell 2013). The updating cost was really tempting (customers received a gift card up to $\$ 250$ to purchase the new version). Why is Apple underpricing its brand new technology? What's the rationale behind this pricing campaign?

In this paper we use an agent-based model (ABM) to study the revenues that can be achieved by a monopolist, which will be referred to as the firm in what follows, in a setup with competition between technologies issued at subsequent dates and volatile consumers. In the last decades several ABMs dealing with innovation and technological change have been proposed (see Adner and Levinthal 2001; Dawid 2006 for a recent review). These papers mainly focus on different patterns of investment strategies for production, heterogeneity of firms with respect to knowledge accumulation, abilities and levels of expertise. The goal is to try to forecast market reactions, considering most of the aspects involved in the decision to invest on incremental innovations, with minor extensions to existing products, or radical ones, with the idea of opening new markets.

\author{
P. Pellizzari \\ Department of Economics, Ca' Foscari University, Venice, Italy \\ e-mail: paolop@unive.it \\ E. Sartori \\ Dept. of Mathematics, University of Padua \\ e-mail: esartori@math.unipd.it \\ M. Tolotti ( $\square)$ \\ Department of Management, Ca' Foscari University, Venice, Italy \\ e-mail: tolotti@unive.it
}


In Deffuant et al. (2005), an ABM to study diffusion of innovation is proposed. In particular, the authors discuss about the rationality of potential adopters and concentrate on their behavioral aspects, in order to show how a market share for a new technology or product forms and evolves over time.

The efficacy of ABMs in the context of diffusion of innovation has been also pointed out in Kiesling et al. (2012). As argued by the authors, although very useful to represent heterogeneous populations, beliefs and network structures, ABMs usually do not take into account competition among technologies. On the first hand, we extend the literature considering two competitive technologies and, on the second hand, we offer an analysis of how market shares are affected by the herding intensity, whose changes can proxy the entry of competitors in the business.

In our model technologies are exogenously given. What we focus on is the problem a firm has to face when deciding to launch new technologies on the market. Indeed, it has to set prices in order to maximize its own revenues. To do so, it forecasts demand and, accordingly, decides the optimal pricing strategy. On the other hand, potential adopters form their expectations about the diffusion of the technology. As a matter of fact, multiple equilibria for the market shares may arise. Using an ABM, we can analyze statistical properties of revenues, prices and market shares and show how, depending on the values of the parameters, completely different outcomes may emerge. Customers are price sensitive, get utility from imitation (herding) and take into account private signals about their willingness to pay (Brock and Durlauf 2001). The two main drivers of our model are, thus, the strength of the imitative behavior, which is anecdotally of great import in the technological market, and the prices set by the firm. We discuss the level of adoption rates at equilibrium as well as the determination of the firm's pricing schedule to maximize revenues.

In Nadal et al. (2005), a one-generation model is studied and a unique price is determined by maximizing the revenues from sales of a unique technology. Motivated by the Apple case described above, we generalize previous work and propose a two-phase model, where the competition among two generations of products makes the picture more involved and realistic. Indeed, facing a two-period game, the firm needs to forecast the emerging adoption rate at the end of the first round, in order to optimally allocate prices for the second period.

We show that, as somewhat expected, the optimal revenues are strongly influenced by the level of imitation on the market. Interestingly and paradoxically, for certain values of the parameters, the firm should optimally give away the update for free, maintaining its market share and boosting revenues.

The paper is organized as follows. In Sect. 2 we describe the decision process of potential adopters (demand) and the optimization scheme of the firm (supply). Section 3 is devoted to the specification of the ABM model. Section 4 addresses the main findings and section Discussion and Conclusion concludes. 


\section{Demand and Supply: Innovators, Updaters and Leapfroggers}

Two sides have to be modeled: a large market of possible adopters (demand) and a firm that sets the prices. We start by the former.

\subsection{The Demand Side}

We consider a population of $N$ possible adopters dealing with the problem of deciding whether to buy an existing technology. In particular, we consider two technologies, $T_{1}$ and $T_{2}$, issued at two subsequent periods. Note that the two technologies are not available to the market at the same time: $T_{1}$ can be bought only in period 1 , whereas technology $T_{2}$ can be bought during period 2 . Moreover, when deciding about the adoption for the first technology, the adopters are not aware of the second generation (or do not take it into account) and can only decide about $T_{1}$. An equilibrium analysis of the decision concerning a single technology has been discussed in the literature, see, for instance Nadal et al. (2005).

During the first period, the agents simultaneously choose whether to adopt $T_{1}$ or not. For each agent, we define the actions $a_{1}(i) \in\{0,1\}$, for $i=1, \ldots, N$, where

$$
a_{1}(i)= \begin{cases}1 & \text { if agent } i \text { decides to adopt } T_{1} \\ 0 & \text { otherwise }\end{cases}
$$

Without loss of generality, we normalize utility from non-adoption to 0 , and set utility from adoption as follows

$$
U_{1}(i)=-p_{1}+q x+\varepsilon_{1}(i)
$$

where $p_{1}>0$ is the price of $T_{1} ; q>0$ is the parameter measuring social utility coming from imitation; $x$ is the expected market share for $T_{1} ;\left(\varepsilon_{1}(i)\right)_{i=1, \ldots, N}$ are i.i.d. random variables with distribution $\eta$. The utility of purchase depends on three summands: an individual noisy term $\varepsilon_{1}(i)$, a positive externality coming from the share of other users who adopt the technology and a negative effect due to the cost of adoption. Each agent compares his own utility of adoption and non-adoption, taking into account costs and social/private benefits. For $N$ fixed, at least one Nash equilibrium in pure strategies $a_{1}^{*}(i)$ exists (see Dai Pra et al. 2013 for details). It can be characterized in terms of an equilibrium market share $x^{(N)}=\frac{1}{N} \sum_{i=1}^{N} a_{1}^{*}(i)$.

Moreover, when the number of possible adopters goes to infinity, any equilibrium market share $x$ is proved to be a solution of the following implicit equation

$$
x=1-\eta\left(p_{1}-q x\right)
$$


If $\eta$ is unimodal, (2) can have one or three solutions depending on the values of $p_{1}$ and $q$. The problem described above belongs to a class of models dating back to the celebrated riot model by Granovetter (see Granovetter 1978), with $x$ representing the fraction of people taking part in a riot. In the presence of herding, a quarrel could morph and escalate into a full blown uprising but, more importantly, the paper makes clear that (equilibrium) results may be extremely sensitive to small changes in the distribution of agents' preferences. This insight is helpful in describing some of the most prominent findings of our model and will be touched upon later.

In period 2 a new technology $T_{2}$ is issued and $T_{1}$ can no longer be adopted; besides, a $T_{1}$ owner can upgrade to $T_{2}$, if desired. Note that, at this stage, there are two different groups of agents: the first consists of leapfroggers (agents who did not adopt $T_{1}$ in the first period), the second of innovators (agents owning $T_{1}$ ). Depending on the group, agents face different utilities. Concerning leapfroggers, we set utility of non-adoption at zero, whereas utility from adoption (denoted by $U_{02}$ ) is

$$
U_{02}(i)=-p_{2 A}+q y+\varepsilon_{2}(i),
$$

where $p_{2 A}$ is the cost for buying $T_{2} ; y$ is the expected market share for $T_{2}$ and $\left(\varepsilon_{2}(i)\right)_{i=1, \ldots, N}$ are i.i.d. terms with distribution $\eta$ representing random terms related to the second technology. Note that $y$ is now defined as $y=y_{A}+y_{U}$, where $y_{A}$ and $y_{U}$ denote, respectively, the proportion of new adopters and updaters in period 2.

Concerning innovators, they have to decide between keeping $T_{1}$ or updating to $T_{2}$. For these groups of agents, the utilities to be compared are

$$
\begin{aligned}
& U_{11}(i)=q\left(x-y_{U}\right)+\varepsilon_{1}(i), \\
& U_{12}(i)=-p_{2 U}+q y+\varepsilon_{2}(i),
\end{aligned}
$$

where $U_{11}$ denotes the utility from maintaining $T_{1}, U_{12}$ the utility from updating, $p_{2 U}$ is the updating cost and $x$ is the market share for $T_{1}$. The term $x-y_{U}$ is the proportion of the aficionados, who prefer to hold $T_{1}$. This is the market share one considers, when evaluating the social utility of being locked in $T_{1}$.

Similarly to period 1 , once the distribution of the noise terms and $p_{2 A}, p_{2 U}$ are fixed, the emergent market share can be computed by solving an implicit system of two equations. In particular, when the number of agents tends to infinity, the equilibrium market shares $y_{A}$ and $y_{U}$ can be formally characterized by the implicit system

$$
\left\{\begin{array}{l}
y_{A}=(1-x)\left[1-\eta\left(p_{2 A}-q y\right)\right] \\
y_{U}=x\left[1-\tilde{\eta}\left(p_{2 U}-q\left(y-\left(x-y_{U}\right)\right)\right)\right],
\end{array}\right.
$$

where $\tilde{\eta}$ denotes the distribution of the random variable $\left(\varepsilon_{2}(i)-\varepsilon_{1}(i)\right)$ conditional on the event $\left\{a_{1}(i)=1\right\}$. If (2) admits a unique solution $x$ and $\eta$ is unimodal, then (5) has one or three solutions. To the best of our knowledge, there is no 
closed-form for the solution of the system (5). Moreover, we are interested in computing market shares when the number of possible adopters is large, but finite. In Sect. 3 we will show how to take advantage of an ABM to compute the emergent market shares.

\subsection{The Supply Side}

The firm chooses prices to maximize revenues, namely the vector $\mathbf{p}=$ $\left(p_{1}, p_{2 A}, p_{2 U}\right)$. Once prices have been set, agents form their demand and market shares emerge. We denote them by the vector $\mathbf{m}=\left(x, y_{A}, y_{U}\right)$, where we drop the dependence on $\mathbf{p}$ for simplicity. Total revenues are

$$
\Pi_{q}(\mathbf{p}, \mathbf{m})=p_{1} x+p_{2 A} y_{A}+p_{2 U} y_{U}=\mathbf{p} \cdot \mathbf{m}^{\prime},
$$

where $q$ is assumed to be exogenous in our model. The strength of imitation $q$ has a very important role in the optimal pricing decision, as shown in the next sections. Notice, moreover, that the objective function (6) depicts a situation in which the revenues in both periods are important as well as interrelated. Indeed, $p_{1}$ has a double role: it (explicitly) determines the revenues of the first period and (implicitly) shapes the picture of the market in the second period. Indeed, one of the outcomes of the first period stage is the identification of innovators and leapfroggers; this distinction clearly affects the second wave of revenues.

\section{The Agent-Based Model}

We now provide details about the construction of the ABM.

\section{Initial set-up}

- We consider all values of $q$ in the grid $\{2.0,2.1, \ldots, 3.9,4\}$. As already said, $q$ will remain the unique exogenously fixed parameter in the model. All the other quantities (prices and shares) will be hereafter determined optimally.

- We fix a three-dimensional grid of values, where triplets of prices $\mathbf{p}=$ $\left(p_{1}, p_{2 A}, p_{2 U}\right)$ are chosen. In the present simulation, we have $p_{1} \in$ $\{1.00,1.05, \ldots, 1.95,2.00\}, p_{2 A} \in\{0.50,0.55, \ldots, 1.45,1.50\}$ and $p_{2 U} \in$ $\{-0.5,-0.4, \ldots, 1.4,1.5\}$.

\footnotetext{
${ }^{1}$ Ranges for $p_{1}, p_{2 A}$ and $p_{2 U}$ have been selected after some preliminary explorations of the location of optimal solutions.
} 
- We consider a finite and large population of agents $(N=1,000)$.

- We simulate $M=50$ different stories for each of the price configurations (triplets) within the grid.

\section{Demand: First period}

- Agent $i$ receives a private signal $\varepsilon_{1}(i)$ : his/her personal view on $T_{1}$. Moreover, all the state variables are fixed at zero (nobody owns the first technology).

- The $N$ agents choose their actions according to utility as in (1). The outcome ${ }^{2}$ is a market share $x^{N}$.

\section{Demand: Second period}

- Agent $i$ receives a private signal $\varepsilon_{2}(i)$ : his/her personal view on $T_{2}$.

- Agents are divided into two groups depending on their action at period 1: leapfroggers or innovators.

- Leapfroggers choose their actions according to (3), whereas innovators rely on (4). The outcome ${ }^{3}$ is a market share $y^{N}=y_{A}^{N}+y_{U}^{N}$.

\section{Supply}

- We evaluate the $M$ revenues for each of the price configurations (triplets) in the grid of values.

- For any given $q$, we select $\mathbf{p}^{*}(q)=\arg \max _{\mathbf{p}} \Pi_{q}(\mathbf{p}, \mathbf{m}(\mathbf{p}))$ as the vector at which the median revenues (over the $M$ simulations) are maximized.

- Then we form, for each $q$, the set $P_{q}$ of price triplets, whose $M$ simulated revenues are not significantly different from the revenues obtained with $\mathbf{p}^{*}(q)$ (using a Wilcoxon test with $5 \%$ significance level). The elements, i.e., prices, of $\mathrm{P}_{q}$ generate revenues that cannot be statistically distinguished from the optimal ones.

\section{Final outcome}

- The set of optimal prices (strategies) $\mathrm{P}_{q}$, together with the emerging market shares and optimal revenues, both in batches of $M$ items, for each $q$ in the grid.

The reader may wonder about the role of the set $\mathrm{P}_{q}$ : recall that prices are discretized and revenues are noisily estimated using simulations. As a result, there may be several different optimal price triplets that should be considered as indistinguishable by the firm as they produce the same revenues. While typically $\mathrm{P}_{q}$ is a "ball" centered at $\mathbf{p}^{*}(q)$, there are values of $q$ for which this set has a non trivial

\footnotetext{
${ }^{2}$ The prevailing market share is obtained simulating repeated adoptions till convergence is reached: in the first round a fraction of $x_{1}$ agents will adopt according to (1) even in the absence of other adopters; in the second round more agents will join based on the current $x_{1}$ and the total fraction rises to $x_{2}$; the process continues till the adopters' share stabilizes at some $t$, i.e., $x_{t}=x_{t+1}$. The prevailing market share is then defined to be $x^{N}=x_{t}$.

${ }^{3}$ The prevailing market shares are obtained as in period 1 , using fictitious rounds of adoptions till convergence is reached.
} 
structure, suggesting that entirely different pricing schedules, supporting diverse market shares, are nevertheless equivalent in terms of revenues. This strategic multiplicity can be appreciated only when $\mathrm{P}_{q}$, as opposed to $\mathbf{p}^{*}(q)$ alone, is analyzed. For a detailed analysis on this aspect, see point 4 in Sect. 4.

\section{Results}

In this section we outline the main findings and the results derived by the ABM procedure.

1. Revenues increase with $q$. The higher is $q$, the higher are the expected revenues, see Fig. 1, left panel. Imitation as measured by $q$ plays a key role in determining the level of the revenues that are almost linearly increasing. It is interesting to decompose the total revenues in the three components due to adoption in period 1 , adoption in period 2 and updating (from $T_{1}$ to $T_{2}$ ) in period 2. Clearly, adoption in the first period brings a major share of revenues, especially when $q$ is high. The income due to adoption in the second period (dashed line) is decreasing with $q$ reflecting, among other things, a mechanical effect: if the share of innovators increases, the group of leapfroggers shrinks. The revenues coming from updaters (dash-dotted line) are small and relatively flat. We will shortly show that this nearly constant stream of revenues is indeed attributable to a rich dynamics connecting the share of adopters and the price of update.

2. The share of updaters peaks at a critical $q$. Figure 1 on the right depicts the (optimal) market shares as functions of $q$. The share of innovators appears
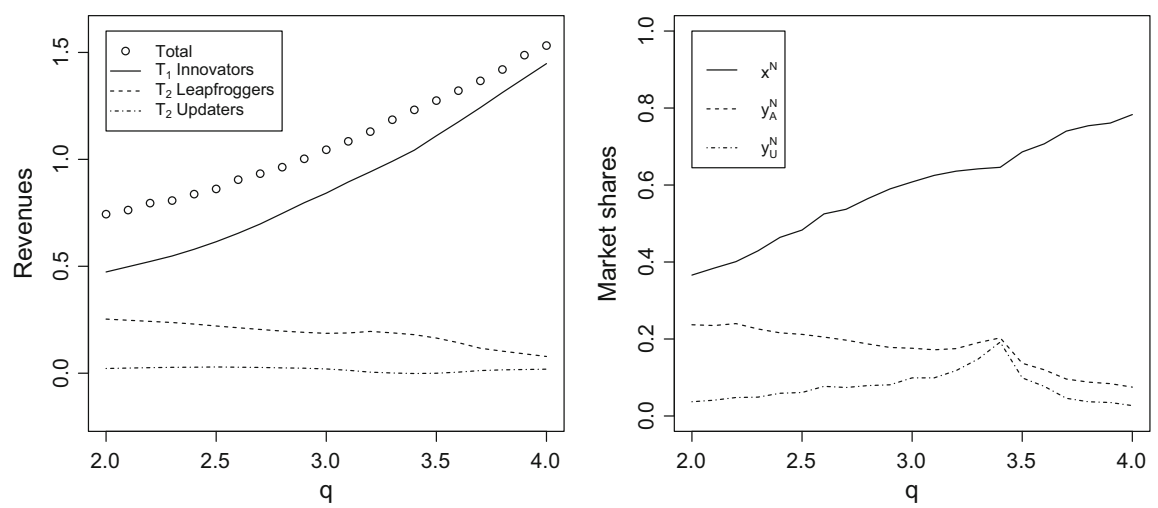

Fig. 1 Left panel: total revenues $\Pi_{q}$ as a function of $q$ (circles). The three lines show the revenues of the first period (solid line) and of the adoption due to leapfroggers (dashed line) and updaters (dash-dotted line), respectively. Right panel: shares of innovators (adopters in period 1), leapfroggers and updaters (adopting or updating in period 2). The three fractions are shown with solid, dashed and dash-dotted lines, respectively 
Fig. 2 Prices for different $q$ : median optimal $p_{1}, p_{2 A}$ and $p_{2 U}$ in the set $\mathrm{P}_{q}$ are shown with circles, triangles and crosses, respectively. Simulation results are smoothed with solid, dashed and dash-dotted lines for added clarity

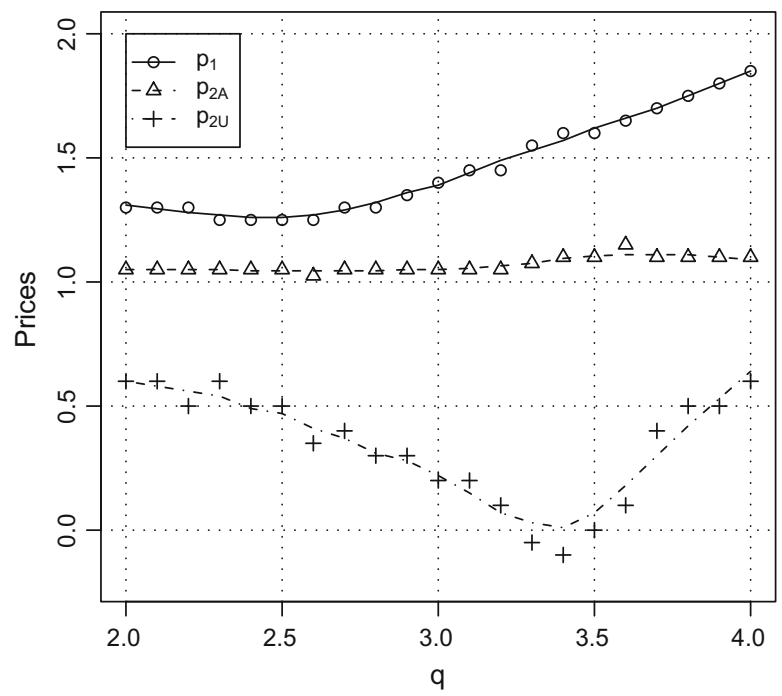

to steadily increase, whereas the fraction of leapfroggers decreases (with a blip around $q \approx 3.4$ ). Interestingly, the share of updaters peaks at the same critical $q$ (dotted line) and reaches $20 \%$ of the customers' base. Intuitively, the increase in the number of updaters helps in pushing more leapfroggers to adopt, thus explaining the temporary deviation from the declining trend of their share (dashed line).

3. The updating price is very low for some values of (critical) $q$. Figure 2 depicts the median optimal prices in the set $\mathrm{P}_{q}$. The price of adoption $p_{1}$ in the first period is nearly constant for $q \leq 2.7$ and increases for higher values of $q$. The price of delayed adoption by leapfroggers is virtually constant across all levels of herding.

The most unexpected and captivating effect is related to the updating price, which is a U-shaped function of $q$. As a matter of fact, updates are very cheap, to say the least, in a range of values of $q$ roughly centered at $q \approx 3.4$, which we previously defined as "critical". Simulations indeed suggest that it may be paradoxically optimal to subsidize updates, being the raw estimates negative in several instances. If we conservatively look at the smoothed dotted line, still we get the insight that updates should optimally be favored with aggressive pricing and massive discounts.

Low updating prices are clearly responsible for the surge in the share of updaters visible at $q \approx 3.4$; in turn, this rise in the number of customers employing $T_{2}$ fosters further (delayed) adoptions by agents who did not adopt $T_{1}$ in the first period.

This provides a strong rationale for the Apple campaign that, actually, offered owners of iPhone 4 a substantial discount to induce them to switch to the new iPhone 5. When externality is sufficiently high (approximately in the interval 
Fig. 3 Median revenues (circles), with their maximum and minimum levels computed considering all the triplets in the set $\mathrm{P}_{q}$

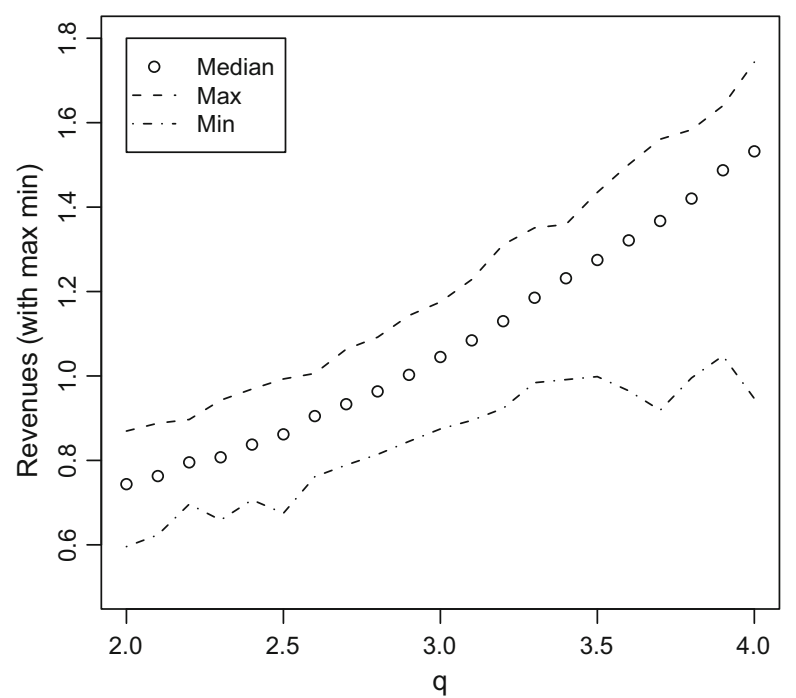

$q \in[3.2,3.6])$, it becomes optimal to give the update for free. This, in essence, is necessary to boost adoptions of leapfroggers, thus maintaining a high market share. Observe that, in the same range of the parameter $q$, the price $p_{2 A}$ of adopting $T_{2}$ stays constant, suggesting that it is preferable to inflate adoptions by enticing old users to upgrade rather then by diminishing the price of the new technology for newcomers. When $q$ is very high ( $q \geq 3.7)$, it is optimal to raise again the updating price, $p_{2 U}$ : the huge externality will sustain new adoptions, regardless of the price.

4. Variability of revenues increase at the critical $q$. Figure 3 shows the total revenues already represented in Fig. 1, left panel (dotted line), but reporting also the maximum/minimum values obtained among the simulations relative to prices in $\mathrm{P}_{q}$ (dashed and dash-dotted lines, respectively). Patently, the dispersion of possible outcomes increases when $q$ exceeds 3.4 and revenues fan out. In particular, for such values of $q$, low values of revenues become significantly frequent. Such variability may be produced by the presence of multiple equilibria in (2) or in the system (5). However, even in the lack of analytical multiplicity of the asymptotic model, sampling fluctuations can give rise to considerable variability of outcomes, as pointed out in Granovetter (1978).

The contour lines of the simulated joint densities of $x^{N}$ and $y_{U}^{N}$ for $q=2.8$ and $q=3.4$ are shown in Fig. 4. The left panel represents market shares for a non-critical $q=2.8$. Typical outcomes nicely crowd in an annular neighborhood of $(0.55,0.10)$ and variability in the shares in this case is only attributable to sample fluctuations of the $N$ idiosyncratic shocks of the agents. In the right panel relative to the critical $q=3.4$, two broad situations are likely to materialize. Selecting an optimal price for that $q$ can produce a continuum of market shares $\left(x^{N}, y_{U}^{N}\right)$ clustered around $(0.7,0.1)$ and $(0.6,0.4)$, approximately. It is 

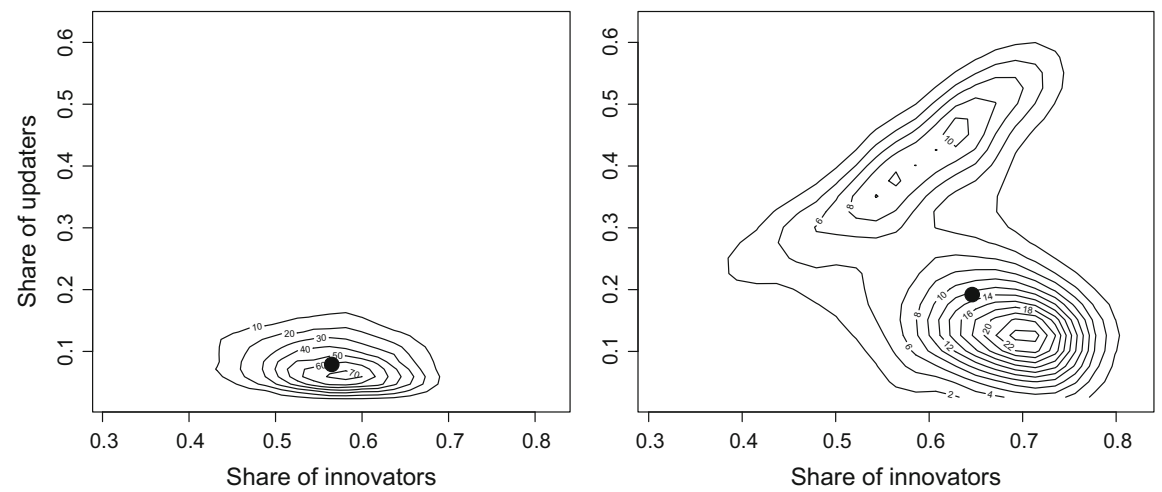

Fig. 4 Contour lines of the simulated joint densities of $x^{N}$ and $y_{U}^{N}$ for $q=2.8$ (left panel) and $q=3.4$ (right panel). Filled dots show the median shares, which are also visible in the right panel of Fig. 1, relative to $q=2.8$ and $q=3.4$

important to stress that, up to some variability discussed previously, all such configurations are equivalent with respect to median revenues.

The pronounced difference between the two panels of Fig. 4 is also indicative of the emergent strategic complexity about the critical value of $q$. The simple attempt to summarize the results of $M$ simulations is hard in this case, as shown by the somewhat puzzling location of the filled point in the right panel, which is barely representative of the richness and complexity of the outcomes.

\section{Discussion and Conclusion}

The findings presented in the previous section allow to deepen the discussion on the rationality of the trade-in campaign of Apple in the US market that was introduced in Sect. 1. Our model singles out that there are values of $q$ in which upgrades should be extremely cheap or even free to boost market shares. In the specific case under scrutiny, at the cost of stretching the model a little bit, we can provide a suggestive description of the following two scenarios: in the first period Apple virtually enjoys a monopolistic position in the market, whereas in the second one the entry of a strong competitor forces Apple to change its pricing campaign. More precisely, we identify period 1 with 2010 and $T_{1}$ with iPhone 4, period 2 with 2012/2013 and $T_{2}$ with iPhone 5. The competitor we are thinking of is Samsung Electronics Co. with its Android operating system. In April 2013, Bloomberg's Adam Satariano described how Apple's quarterly profit was projected to shrink for the first time in a decade, especially due to this new competitor (see Satariano 2013).

As a first approximation, we model the competition with a simple decrement in the externality parameter $q$, induced by the entry of the competitor. 
Let's assume the value of $q$ for Apple in 2010, before the arrival of Samsung, is high (say, close to $q=4$ ). This value is justified by the remarkable loyalty Apple's fans have always demonstrated to the company. As a consequence, Apple is in a very strong position and can put in place a pricing campaign in which prices are very high, without suffering a decrease in revenues. Once the competitor enters the market (during 2012/2013), a sudden decrease in $q$ may be expected. Suppose indeed, that $q$ falls to about $q=3.5$. The picture is, thus, different: Apple must revise its aggressive pricing policy in order to maintain its market share. Now, our results suggest that the optimal policy is to give away the update for free. Our model, although very stylized, supports the rationality of an aggressive reduction in the price of updates.

We would like to mention a second feature of the trade-in program implemented by Apple, which is de facto equivalent to a further price reduction for the upgrade: Apple launched the iPhone 5 together with a new release of the iPhone operating system iOS7, which was believed by many users to be the cause of a deterioration in the performances of iPhone 4. It was possible to overcome such "technical" problem by replacing the battery at a cost of about $\$ 70$, or just upgrading to the new iPhone 5 with a lump-sum of about $\$ 99$ (thanks to the high discount due to the trade-in program). We do not know whether this was a measure intentionally planned to pursue the company's own objectives (see Rampell 2013) but, in practice, these events additionally increased the cost of keeping the iPhone 4 .

On a different note, our model vividly illustrates a feature of emerging equilibria of interacting agents in binary decisions models. Even when a formal analysis rules out the existence of multiplicity, the sampling variability inherent in any simulation can lead to markedly different results. For some values of $q$, which approximate what would be described as a (near) "tangency" in Granovetter (1978), this is exactly what happens in our setup. The relevance is twofold: on the first hand, outcomes are affected by unavoidable levels of uncertainty, despite the fact of being based on optimal decisions, and the firm should be aware of this strategic unpredictability; on the other hand, such sensitivity does not disappear even if an analytical model is at hand. For instance, existence and uniqueness of an equilibrium do not imply that finite size simulations will converge to the unique equilibrium.

We assume in the model that the individual shocks related to the preferences of the agents are independent. This is a limitation of the present treatment as it is likely that $\varepsilon_{1}$ is orthogonal to $\varepsilon_{2}$ only if the two competing technologies are radically different. In many realistic cases, subsequent waves of products may carry relatively minor technical changes or moderate improvements in usability. In these cases, the noise terms would be (strongly) positively correlated. Hence, our model may be more suited to describe 
situations in which fundamental developments have been introduced or in the presence of a notable shift with respect to the past paradigms.

Further generalizations of the model may also remove the assumption that the firm commits itself to fix prices in the first period. Clearly, the price of getting (or updating to) $T_{2}$ needs not to be disclosed to customers in the first period but an alternative course of action would suggest to determine $p_{2 A}$ and $p_{2 U}$ after the market share $x^{N}$ emerges. Instead of being unconditionally worked out together with $p_{1}$, the selection of prices for $T_{2}$ should be conditional on the realized market share, in a backward-like fashion resembling Bellman dynamic programming principle. In this respect, the revenues of the present model can be interpreted as lower bounds for a conditional pricing strategy, which may result in additional marginal excess gains.

Acknowledgements The authors thank Marco LiCalzi for insightful comments and discussions and participants to seminars held at XXXVII AMASES meeting, Stresa; Collegio Carlo Alberto, Torino and Alpen-Adria Universität, Klagenfurt. We received financial support from MIUR under grant "Robust decision making in markets and organizations" (PRIN20103S5RN3).

\section{References}

Adner R, Levinthal D (2001) Demand heterogeneity and technology evolution: implications for product and process innovation. Manag Sci 47(5):611-628

Brock WA, Durlauf S (2001) Discrete choice with social interactions. Rev Econ Stud 68(2):235260

Dai Pra P, Sartori E, Tolotti M (2013) Strategic interaction in trend-driven dynamics. J Stat Phys 152:724-741

Dawid H (2006) Agent-based models of innovation and technological change. In: Tesfatsion L, Judd KL (eds) Handbook of computational economics, vol 2. Elsevier, Amsterdam, pp 12351272

Deffuant G, Huet S, Amblard F (2005) An individual-based model of innovation diffusion mixing social value and individual benefit. Am J Sociol 110(4):1041-1069

Granovetter M (1978) Threshold models of collective behavior. Am J Sociol 83(6):1420-1443

Kiesling E, Gunther M, Stummer C, Wakolbinger LM (2012) Agent-based simulation of innovation diffusion: a review. Cent Eur J Oper Res 20(2):183-230

Nadal J, Phan D, Gordon M, Vannimenus J (2005) Multiple equilibria in a monopoly market with heterogeneous agents and externalities. Quant Financ 5(6):557-568

Rampell C (October 29, 2013) Cracking the apple trap. The New York Times Magazine

Satariano A (April 22, 2013) Apple profit probably fell amid growth slowdown for iphone. Bloomberg.com 